Herz 2019 • 44:1-3

https://doi.org/10.1007/s00059-018-4771-6

(c) Springer Medizin Verlag GmbH, ein Teil von Springer Nature 2018

CrossMark

\section{H. J. Rupprecht ${ }^{1} \cdot$ R. Erbel $\left.\right|^{2} \cdot$ R. Dörr ${ }^{3}$}

${ }^{1}$ II. Medizinische Klinik, Innere Medizin - Kardiologie - Intensivmedizin, GPR Klinikum Rüsselsheim Rüsselsheim, Deutschland

${ }^{2}$ Institut für Medizinische Informatik, Biometrie und Epidemiologie, Universitätsklinikum Essen, Universität Duisburg-Essen, Essen, Deutschland

${ }^{3}$ Praxisklinik Herz und Gefäße, Dresden, Deutschland

\title{
Wie alles begann
}

Die bahnbrechende Entwicklung, die Andreas Grüntzig mit der ersten perkutanen transluminalen Koronarangioplastie (PTCA) im September 1977 bei einem Patienten mit stabiler Angina und hochgradiger proximaler $\mathrm{LAD}$ („left anterior descending")-Stenose in Zürich einleitete, markiert den Beginn der modernen interventionellen Kardiologie $[1,2]$. Zunächst war der Einsatz dieser neuen Methode auf Patienten mit stabiler koronarer Herzkrankheit (KHK) begrenzt. Tatsächlich waren seinerzeit heute kaum noch vorstellbare ungünstige Bedingungen gegeben. Ein Führungskatheter, noch ohne spezielle Vorbiegung für rechte und linke Koronararterie, musste über eine 10-FSchleuse vorgebracht werden, verlor im Blutstrom schnell seine Konfiguration und stand oftmals frei schwebend und senkrecht vor dem Koronarostium. Das Armamentarium bestand darüber hinaus aus steifen, noch handgefertigten großlumigen Ballonkathetern, die ohne Hilfe eines Führungsdrahts (Overthe-wire-Systeme gab es noch nicht) in das Kranzgefäß manövriert werden mussten. Nur ein kleines an die Ballonkatheterspitze fixiertes Drahtsegment erlaubte eine gewisse Hilfe bei der Sondierung des Gefäßes. Auch die heute verwendeten Handmanometer waren noch nicht entwickelt, der erforderliche Ballondruck musste mit Hilfe aufwendiger externer Apparaturen aufgebaut werden. Unter diesen Bedingungen und in Ermangelung potenter antithrombotischer Konzepte war die Erfolgsrate mäßig (ca. $70 \%$ für LAD und rechte Koronararterie [„right coronary artery", RCA], $50 \%$ für Ramus circumflexus [RCX]). Das Ergebnis war durch Dissek- tionen, thrombotische Komplikationen und den sog. "Recoil“ nach heutigen Maßstäben oft suboptimal. Da Stents noch nicht zur Verfügung standen, war das Risiko eines akuten oder subakuten Gefäßverschlusses allgegenwärtig und im Langzeitverlauf eine Restenoserate von $30-50 \% \mathrm{zu}$ erwarten.

Nach Teilnahme an einem von Andreas Grüntzig organisierten Kurs in Zürich hatte Jürgen Meyer bereits 1978 frühe Erfahrungen mit der PTCA bei Patienten mit stabiler Angina in der Aachener Klinik gesammelt. Ein klinisches Dilemma war Anlass für den ersten Einsatz der PTCA bei einer Patientin mit instabiler Angina pectoris. Bei der Patientin war wegen eines Tumorleidens eine dringliche Operation in der Allgemeinchirurgie anberaumt worden. Wegen einer präoperativ sich entwickelnden refraktären instabilen Angina war aber an einen Eingriff nicht zu denken. Eine Koronarangiographie zeigte eine hochgradige proximale Stenose der LAD. In dieser Zwangslage führte Jürgen Meyer eine Ballondilatation durch. Der Zustand der Patientin konnte so stabilisiert und die Operation anschließend durchgeführt werden [3]. Ermutigt durch diesen positiven Verlauf, konnten bald Ergebnisse bei weiteren Patienten in dieser Indikation berichtet werden $[4,5]$. Da die Troponintestung zum damaligen Zeitpunkt noch nicht zur Verfügung stand, dürfte bei einem mutmaßlich großen Teil der seinerzeit als ,instabil“" klassifizierten Patienten nach heutiger Nomenklatur ein NSTEMI („non ST segment elevation myocardial infarction“) vorgelegen haben. Jürgen Meyer war auch der Erste, der schon sehr früh kritisch auf die besonderen Probleme der PTCA unter den Bedingungen des a prio- ri thrombotischen Milieus bei akutem Koronarsyndrom im Vergleich zur stabilen Situation hinwies, wie z. B. die geringere primäre Erfolgsrate, die höhere Rate an Akutkomplikationen und auch eine höhere Restenoserate [4,5]. Dies bestätigte sich auch im Langzeitverlauf [6]. Es bedurfte großer Standhaftigkeit, den eingeschlagenen Weg gegen Widerstände und trotz unvermeidbarer Rückschläge, aber vom damaligen Klinikchef Sven Effert bestärkt, fortzusetzen.

Als weitere Entwicklung zeichnete sich ab, dass die intrakoronaren Thromben, die von Pathologen postmortal bei Infarktpatienten gesehen wurden, nicht als postmortale Artefakte anzusehen waren. Vielmehr konnte DeWood zeigen, dass ein frischer thrombotischer Verschluss einem Infarkt ursächlich zugrunde lag [7]. Diese Erkenntnis eröffnete den Weg zur Einführung der Thrombolysetherapie, wobei die intrakoronare mit der intravenösen Gabe konkurrierte [8]. Rentrop war es auch, der erstmals einen während einer Koronarangiographie akzidentell in ein Kranzgefäß verschleppten Thrombus mit einem Draht rekanalisierte. Dies war die erste mechanische Rekanalisation eines Kranzgefäßes [9]. Bei den im sog. Lysedienst behandelten Infarktpatienten fiel auf, dass nach einer erfolgreichen Lyse nahezu regelhaft hochgradige Stenosen im Infarktgefäß verblieben, die ihrerseits nicht selten Kristallisationspunkt für erneute thrombotische Verschlüsse waren. Jürgen Meyer hat, ausgehend von diesen Beobachtungen, mit seiner Aachener Arbeitsgruppe die PTCA erstmals bei Patienten mit akutem Infarkt (heute STEMI) eingesetzt $[10,11]$. Nach ersten erfolgversprechen- 
den Behandlungen wurde die PTCA bei Patienten mit ST-Hebungs-Infarkt in der klinischen Routine etabliert. In weiteren, auch randomisierten Studien konnte der Vorteil dieser mechanischen Reperfusionsbehandlung, nämlich eine verbesserte linksventrikuläre (LV-) Funktion und geringere Reverschlussrate, belegt werden [12]. Die bis dahin noch häufig begleitende oder vorausgehende Thrombolysetherapie wurde im weiteren Verlauf zunehmend zugunsten der reinen mechanischen Reperfusion (direkte perkutane koronare Intervention [PCI]) verlassen.

Die höchste Letalität (bis zu 80 \%) wiesen Patienten im infarktbedingten kardiogenen Schock auf. Auch nach Einführung der Thrombolysetherapie verstarben noch mehr als $50 \%$ der Patienten. Im Januar 1981 war eine 72-jährige Patientin mit akutem Hinterwandinfarkt ins Aachener Klinikum eingeliefert worden. Die dominante rechte Kranzarterie war im mittleren Drittel der Pars descendens verschlossen, die LAD war beiZ. $n$. früherem Vorderwandinfarkt proximal, aber chronisch verschlossen. Eine intrakoronare Lyse mit Streptokinase war zunächst erfolgreich, d.h. führte zu einer Reperfusion der RCA sowie zur Darstellung einer guten Kollateralisierung zur LAD. Wahrscheinlich war es durch den akuten Verschluss der RCA auch zu einem Reinfarkt im Vorderwandbereich gekommen, im Sinne eines ,infarction at a distance“. Nach zunächst stabilem Verlauf entwickelte die Patientin etwa $30 \mathrm{~h}$ später einen progredienten kardiogenen Schock. Der damals auf der Intensivstation diensthabende Kardiologe (und heutige Mitherausgeber von Herz) Rolf Dörr erinnert sich: „Die Patientin rutschte trotz maximaler Katecholamindosen immer weiter in den kardiogegen Schock bis zu einem systolischen Druck von $60 \mathrm{~mm} \mathrm{Hg}$. Es war eine verzweifelte Situation."Gegen Mitternacht wurde Jürgen Meyer hinzugerufen. Eine erneut durchgeführte Koronarangiographie zeigte eine nur noch gering perfundierte und an der vorherigen Verschlussstelle höchstgradig stenosierte rechte Kranzarterie. Die vorher gut sichtbaren Kollateralen zur chronisch verschlossenen $\mathrm{LAD}$ waren kaum noch erkennbar. In dieser ausweglosen Situati- on entschied Jürgen Meyer, eine Ballondilatation zu versuchen. Es gelang, den Ballonkatheter in den Bereich der subtotalen Stenose vorzuführen. Nach mehrfacher Dilatation konnte eine befriedigende Aufdehnung der zugrunde liegenden RCA-Stenose und eine vollständige Wiederherstellung des Blutflusses in der RCA erreicht werden. Insbesondere wurde auch die bedrohte Kollateralversorgung zur LAD wiederhergestellt. In der Folge kam es zu einer raschen Stabilisierung der hämodynamischen Situation. Die erfolgreiche Intervention ermöglichte der Patientin noch ein Überleben für mehrere Jahre. Das Fazit dieser „Firstin-man"-Behandlung eines kardiogenen Schocks mit Hilfe der direkten PTCA wurde in der nachfolgenden Publikation von Jürgen Meyer [13] wie folgt formuliert: „... PTCA of residual fixed stenosis can provide considerable improvement in myocardial perfusion and ventricular performance, and can reverse AMI cardiogenic shock." In den folgenden Jahren entwickelte sich die direkte PTCA zunehmend zum Goldstandard der Therapie des kardiogenen Schocks. Dennoch dauerte es seit der Erstbeschreibung noch nahezu zwei Jahrzehnte, bis nach Publikation eines internationalen Registers und einer randomisieren Studie letzte Zweifel an dem Behandlungskonzept ausgeräumt waren [14, 15].

Andreas Grüntzig hatte in der frühen Phase die Anwendung der PTCA auf Patienten mit stabiler KHK fokussiert und stand dem Einsatz bei akutem Koronarsyndrom zunächst kritisch gegenüber. Es ist das große Verdienst von Jürgen Meyer, das Potenzial dieser neuen Technologie für Patienten mit allen Formen des akuten Koronarsyndroms als Erster erkannt und auf der Grundlage sorgfältiger und systematischer klinischer Forschung die PTCA in der klinischen Routine etabliert zu haben. Während heute, auch mehr als 40 Jahre nach der ersten PTCA durch Andreas Grüntzig, noch um den Stellenwert bei der stabilen KHK gerungen wird (wie kürzlich in der viel diskutierten und öffentlichkeitswirksamen ORBITAStudie), ist die PCI bei allen Formen des akuten Koronarsyndroms weltweit integraler Bestandteil und Klasse-I-Indikation in allen Leitlinien. Unzweifelhaft hat diese Behandlungs-Methode zahllosen Patienten, insbesondere bei akuten Koronarsyndromen, ein Weiterleben ermöglicht oder eine frühe Invalidität verhindert. Aus heutiger Sicht war die Entwicklung der PTCA durch Andreas Grüntzig eine Jahrhunderterfindung. Für das akute Koronarsyndrom, von der instabilen Angina bis hin zum Infarkt mit kardiogenem Schock, wurde dieser Schlüsseltechnologie aber von Jürgen Meyer entscheidend der Weg bereitet.

Diese Ausgabe von Herz ist Herrn Professor Jürgen Meyer anlässlich seines 80. Geburtstages für seine Pionierleistungen bei der Behandlung des akuten Koronarsyndroms gewidmet.

\section{Korrespondenzadresse}

Prof. Dr. med. H. J. Rupprecht

II. Medizinische Klinik, Innere Medizin -

Kardiologie - Intensivmedizin, GPR Klinikum

Rüsselsheim

August-Bebel-Str. 59, 65428 Rüsselsheim,

Deutschland

hj.rupprecht@gp-ruesselsheim.de

Interessenkonflikt. H.J. Rupprecht, R. Erbel und R. Dörr geben an, dass kein Interessenkonflikt besteht.

\section{Literatur}

1. Grüntzig A, Hirzel H, Goebel N, Gattiker R et al (1978) Percutaneous transluminal dilatation of chronic coronary stenoses. First experiences. Schweiz Med Wochenschr 108:1721-1723

2. Grüntzig AR, Senning A, Siegenthaler WE (1979) Nonoperative dilatation of coronary-artery stenosis: percutaneous transluminal coronary angioplasty. NEng J Med 301:61-68

3. Meyer J, Messmer BJ, Reifferscheid M (1981) Preoperative treatment of severe coronary stenosis with the balloon catheter in patients with operable carcinomas (author's transl). Dtsch Med Wochenschr 106:1366-1369

4. Meyer J, Schmitz H, Erbel R, Kiesslich T et al (1981) Treatment of unstable angina pectoris with percutaneous transluminal coronary angioplasty (PTCA). Cathet Cardiovasc Diagn 7:361-371

5. Meyer J, Schmitz HJ, Kiesslich T, Erbel Ret al (1983) Percutaneous transluminal coronary angioplasty in patients with stable and unstable angina pectoris: analyses of early and late results. Am Heart J 106:973-980

6. RupprechtHJ,BrenneckeR, Kottmeyer M,Bernhard $\mathrm{G}$ (1990) Short and longterm outcome after PTCA in patients with stable and unstable angina. Eur Heart J 11(11):964-973

7. DeWood MA, Spores J, Notske R, Mouser LT et al (1980) Prevalence of total coronary occlusion during the early hours of transmural myocardial infarction. NEngl J Med 303(16):897-902

8. Rentrop P, Blanke $\mathrm{H}$, Karsch KR, Kaiser $\mathrm{H}$ et al (1981) Selective intracoronary thrombolysis in 
acute myocardial infarction and unstable angina pectoris. Circulation 61(2):307-317

9. Rentrop P, De Vivie ER, Karsch KR, Kreuzer H (1978) Acute coronary occlusion with impending infarction as an angiographic complication relieved by a guide-wire recanalization. Clin Cardiol 1(2):101-106

10. Meyer J, Merx W, Schmitz H, Erbel R et al (1982) Percutaneous transluminal coronary angioplasty immediately after intracoronary streptolysis of transmural myocardial infarction. Circulation 66:905-913

11. Meyer J, Merx W, Schweizer P, Dörr R et al (1982) Selective intracoronary lysis and transluminal coronary dilatation as an immediate measure in acute myocardial infarct. Verh Dtsch Ges Herz Kreislaufforsch 48:157-165

12. Erbel R, Pop T, Henrich KJ, von Olshausen $\mathrm{K}$ et al (1986) Percutaneous transluminal coronary angioplasty after thrombolytic therapy: a prospective controlled randomized trial. J Am Coll Cardiol 8:485-495

13. Meyer J, Merx W, Dörr $\mathrm{R}$, Lambertz $\mathrm{H}$ et al (1982) Successful treatment of acute myocardial infarction shock by combined percutaneous transluminal coronary recanalization (PTCR) and percutaneous transluminal coronary angioplasty (PTCA). Am Heart J 103(1):132-134

14. Lee L, Erbel R, Brown TM, Laufer N et al (1991) Multicenter registry of angioplasty therapy of cardiogenic shock: initial and longterm survival. J Am Coll Cardiol 17(3):599-603

15. Hochman JS, Sleeper LA, Webb JG, Sanborn TA et al (1999) Early revascularization in acute myocardial infarction complicated by cardiogenic shock. Shockinvestigators. NEngl J Med 341(9):625-634

\section{Rheuma birgt hohes Risiko für Begleiterkrankungen}

Aktuelle Studie belegt Gefahr der Unterversorgung

Menschen mit Gelenkrheuma haben oft auch ein erhöhtes Risiko für eine Reihe anderer Erkrankungen. Das ist das Ergebnis einer auf deutschen Krankenkassendaten basierenden Studie, die im Journal of Rheumatology erschienen ist. Sowohl Herz-Kreislauf-Erkrankungen, als auch Erkrankungen des Bewegungsapparats, der Lunge oder der Psyche sind demnach unter Rheumapatienten häufiger als unter gleichaltrigen Vergleichspersonen ohne rheumatoide Arthritis.

Für ihre Studie haben Mediziner und Statistiker vom Department für Versorgungsforschung der Universität Oldenburg und dem Deutschen Rheuma-Forschungszentrum Berlin (DRFZ) die Krankenkassendaten von insgesamt knapp 97.000 Rheumapatienten und 484.000 Vergleichspersonen mit ähnlichem Alters- und Geschlechtsprofil analysiert und 26 Begleiterkrankungen sogenannte Komorbiditäten - erfasst. Wie sich zeigte, leiden Patienten mit einer Rheumadiagnose besonders häufig auch unter Bluthochdruck (62,5 Prozent), Arthrose (44 Prozent), hohen Blutfettwerten (40 Prozent) und Depressionen (31,8 Prozent). „Diese Krankheiten sind zwar auch bei den Vergleichspatienten die häufigsten", sagt Dr. med. Katinka Albrecht vom Programmbereich Epidemiologie und Versorgungsforschung des DRFZ, wo das Verbundprojekt koordiniert wurde. Die absoluten Zahlen seien jedoch in der Vergleichsgruppe viel niedriger: So sei etwa das Arthroserisiko in der Normalbevölkerung nur rund halb so hoch wie bei Rheumapatienten, Depressionen seien um rund ein Drittel seltener.

Ein deutlicher Unterschied zeigt sich auch bei der Osteoporose: Während rund jeder vierte Rheumapatient unter dem Verlust an Knochensubstanz leidet, ist es bei den Vergleichspatienten nur knapp jeder zehnte.

\section{Befragung betroffener Versicherter}

Die Krankenkassendaten wurden ergänzt um eine Befragung einer Zufallsstichprobe betroffener Versicherter. Es zeigte sich, dass die zusätzliche Krankheitslast nicht ohne Folgen bleibt: Je mehr Begleiterkrankungen die Patienten aufweisen, desto höher sind ihre gesundheitlichen Einschränkungen und desto seltener befinden sie sich in Behandlung bei einem spezialisierten Rheumatologen. "Es ist naheliegend, dass bei vielen Begleiterkrankungen der Hausarzt die führende
Rolle übernimmt und gerade ältere Patienten nicht mehr für jede Komorbidität einen Spezialisten aufsuchen", mutmaßt Albrecht.

\section{Medikamentöse Wechselwirkungen und andere Komplikationen}

Gerade multimorbide Patienten sollten jedoch besonders intensiv rheumatologisch betreut werden. Denn mit der Zahl der Krankheiten und der eingenommenen Medikamente steigt das Risiko von medikamentösen Wechselwirkungen und anderen Komplikationen deutlich an. „Dass gerade diese Patientengruppe eine schlechtere rheumatologische Behandlung erhält, ist absurd und muss geändert werden", sagt Prof. Dr. med. Hanns-Martin Lorenz, Präsident der Deutschen Gesellschaft für Rheumatologie, Leiter der Sektion Rheumatologie am Universitätsklinikum Heidelberg und medizinisch-wissenschaftlicher Leiter des ACURA-Rheumazentrums Baden Baden. Als zentrale Ansprechpartner sei es vor allem Aufgabe der Hausärzte, Patienten mit Verdacht auf Rheuma auf die Möglichkeiten und die Notwendigkeit einer gezielten Therapie aufmerksam zu machen und an einen internistischen Rheumatologen zu überweisen. Denn bei frühzeitiger Diagnose und konsequenter anti-entzündlicher Therapie ließen sich die Symptome in vielen Fällen beherrschen und Folgeschäden wie dauerhafte Bewegungseinschränkungen vermeiden.

Literatur:

The Journal of Rheumatology 2019

DOI: https://doi.org/10.3899/jrheum.180668

\section{Stephanie Priester, Pressestelle Deutsche Gesellschaft für Rheumatologie (DGRh)}

